\title{
Determination of Electrophysiologically Moderate and Severe Carpal Tunnel Syndrome: Ultrasonographic Measurement of Median Nerve at the Wrist
}

\author{
Chanwit Phongamwong, MD, MS, Narathorn Soponprapakorn, MD, Wipoo Kumnerddee, MD \\ Department of Rehabilitation Medicine, Phramongkutklao Hospital and Phramongkutklao \\ College of Medicine, Bangkok, Thailand
}

\begin{abstract}
Objective To establish the cutoff value of cross-sectional area (CSA) of the median nerve at the wrist, for determination of electrophysiologically moderate and severe carpal tunnel syndrome (CTS).

Methods The prospective study was conducted among patients suspected of having CTS. A total of 106 patients (185 symptomatic wrists) received nerve conduction study (NCS) and ultrasonography. To establish a cutoff value, various diagnostic properties were calculated across a range of the CSA.

Results A mean \pm standard deviation of CSA of the median nerve of normal and mild, moderate and severe CTS was 9.4 $\pm 2.1,12.0 \pm 2.7,13.8 \pm 4.7$, and $15.4 \pm 4.1 \mathrm{~mm}^{2}$, respectively. The positive relationship between CTS severities and CSA was observed $\left(\mathrm{r}_{\mathrm{s}}=0.56\right)$. A $14 \mathrm{~mm}^{2}$ CSA had sufficient power to rule in moderate and severe CTS, with a specificity of $91.4 \%$ and sensitivity of $42.3 \%$. In addition, it showed a post-test probability (positive predictive value) of $86.3 \%$ as against a pre-test probability of $56.2 \%$.

Conclusion Patients who had $\geq 14 \mathrm{~mm}^{2}$ of median nerve CSA had very high probability of moderate to severe CTS.
\end{abstract}

Keywords Median nerve, Carpal tunnel syndrome, Ultrasonography, Electrophysiology

\section{INTRODUCTION}

Carpal tunnel syndrome (CTS) is the most common upper limb compressive neuropathy, affecting $2.7 \%$ of the general population [1]. The diagnosis of CTS is usually established by history and physical examination. Electrophysiological studies help in CTS diagnosis and severity grading $[2,3]$. Another useful diagnostic tool is ultrasonography, which is painless, rapid and with reduced cost. The common and reproducible finding in compressive

Received September 26, 2016; Accepted December 13, 2016

Corresponding author: Chanwit Phongamwong

Department of Rehabilitation Medicine, Phramongkutklao Hospital and Phramongkutklao College of Medicine, 315 Ratchawithi road, Bangkok 10400, Thailand. Tel: +82-66-2-644-8995, Fax: +82-66-2-644-8995, E-mail: chanwit.pmrpmk@hotmail.com

ORCID: Chanwit Phongamwong (http://orcid.org/0000-0002-5562-8175); Narathorn Soponprapakorn (http://orcid.org/0000-0002-5296-5539); Wipoo Kumnerddee (http://orcid.org/0000-0002-9317-5779).

@ This is an open-access article distributed under the terms of the Creative Commons Attribution Non-Commercial License (http://creativecommons.org/ licenses/by-nc/4.0) which permits unrestricted noncommercial use, distribution, and reproduction in any medium, provided the original work is properly cited. Copyright (c) 2017 by Korean Academy of Rehabilitation Medicine 
neuropathies is nerve enlargement just proximal to the site of entrapment $[4,5]$. Moreover, it detects structural abnormalities at the wrist in patients having CTS [6-8].

According to a paper of the American Association of Neuromuscular and Electrodiagnostic Medicine in 2012, ultrasonography measurement of the median nerve cross-sectional area (CSA) used to determine the median nerve enlargement at the wrist is accurate for the diagnosis of CTS [9]. Cutoff values of 9 or $10 \mathrm{~mm}^{2}$ were suggested as single diagnostic criterion in most previous studies [4,10-14]. Interestingly, several studies reveal that there is a positive correlation between the median nerve CSA and electrophysiological severities of CTS $[15,16]$. However, no well-designed study has been conducted for diagnosis of electrophysiologically moderate and severe CTS, which are to be considered for surgical treatment [17]. Therefore, the primary objective of the present study was to establish a cutoff of the median nerve CSA at the wrist, to determine electrophysiologically moderate and severe CTS.

\section{MATERIALS AND METHODS}

The present research was a prospective study conducted between May 2014 and October 2015. Eligibility criteria included Thai adults (18 years old or over) presenting with sensory symptoms (numbness and/or tingling) in at least 2 digits among $1,2,3$, and 4 for at least 1 month
[2], and able to communicate in Thai. Patients with clinically or electrophysiologically suspected other neurologic diseases such as polyneuropathy, cervical radiculopathy, or proximal median neuropathies, were excluded in advance. In addition, symptomatic wrists were excluded if they had deformities, space occupying lesions, history of steroid injection or wrist surgery (Fig. 1). Participants were enrolled prospectively, and written consent to participate in the present study was obtained. The study protocol was approved by the Institutional Review Board before participant enrollment (IRBRTA 085/2557).

\section{Nerve conduction studies}

A single physiatrist administered standard nerve conduction study (NCS) to all participants. The routine NCS for diagnosing CTS comprised 14-cm antidromic sensory median and ulnar peak latencies, 8- $\mathrm{cm}$ median and ulnar compound muscle action potential, distal motor latencies, and nerve conduction velocity (Medelec Synergy T5EP model; Oxford Instruments, Oxfordshire, UK). The CTS was diagnosed by following the protocol when 14$\mathrm{cm}$ antidromic sensory median peak latency was more than $3.6 \mathrm{~ms}$, or a $14-\mathrm{cm}$ antidromic sensory peak latency difference of the median-ulnar ring finger (ring-diff) was equal to or more than $0.5 \mathrm{~ms}[2,18]$. The NCS results were classified into four groups: no, mild, moderate, and severe CTS, following the recommendation of Stevens [19]. To establish the cut-off for moderate and severe CTS, par-

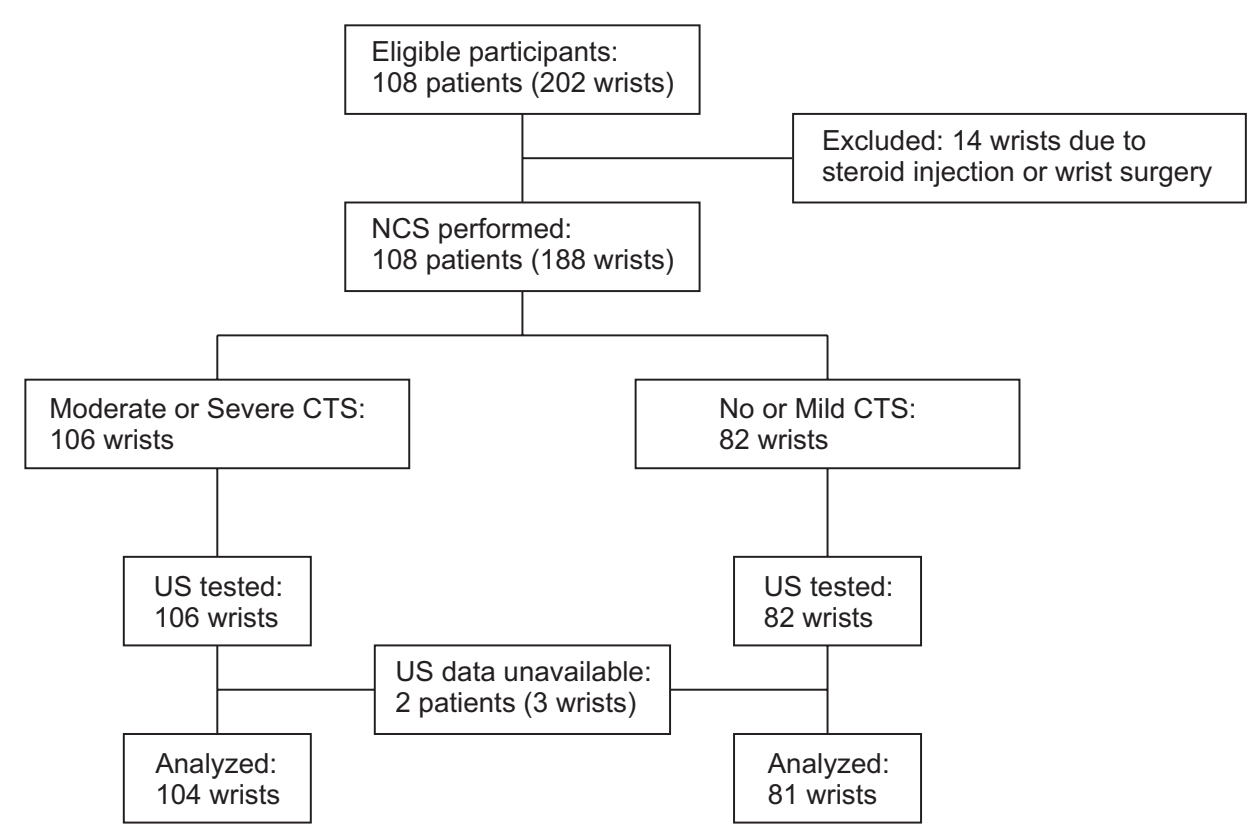

Fig. 1. Flow diagram of the study. NCS, nerve conduction study; CTS, carpal tunnel syndrome; US, ultrasonography. 
ticipants were further categorized into two groups: $0=$ no and mild CTS; $1=$ moderate and severe CTS.

\section{Ultrasonography}

On the same day of NCS, all participants received ultrasonography (US) examination by a physiatrist who had 2 years of experience in neuromuscular US, and was blinded from NCS results. The examinations were conducted with a 14-16 MHz linear array transducer (ProSound Alpha 7; Hitachi-Aloka Medical, Tokyo, Japan). Participants were examined in the sitting position, with palm upward and resting on a pillow across the lap [5]. The CSA $\left(\mathrm{mm}^{2}\right)$ of the median nerve of the symptomatic wrist was imaged and measured at the distal wrist crease (proximal to the carpal tunnel inlet) [4] using direct trace with electronic calipers, and considering the hyperechoic sheath as the margin [10]. The area of each wrist was averaged from three separate examinations. To assess inter-rater reliability, some participants were randomly sampled to be re-examined by another physiatrist with the same protocols and on the same day as the first examination; this physiatrist was blinded from both NCS and US results of the previous examiner.

\section{Statistical analysis}

The inter-rater reliability of US was assessed by intraclass correlation coefficients $(2,1)$. Next, the overall diagnostic value of US was evaluated by the area under the receiver operator characteristics (ROC) curve. Spearman rank correlation coefficient was used to assess the relationship between CSA and electrophysiological re-

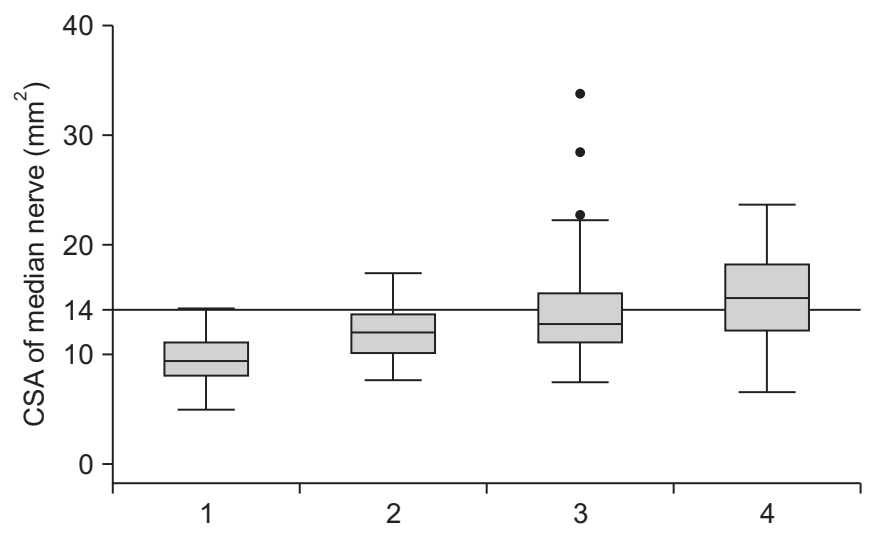

Fig. 2. Correlation between median nerve CSA (crosssectional area; $\mathrm{mm}^{2}$ ) and carpal tunnel syndrome severities ( $1=$ no, $2=$ mild, $3=$ moderate, $4=$ severe). sults. The data was analyzed for calculating sensitivity, specificity, positive predictive value (PPV), and negative predictive value (NPV) across a range of the CSA (10 and $15 \mathrm{~mm}^{2}$ in $1 \mathrm{~mm}^{2}$ increments).

\section{RESULTS}

A total of 108 eligible patients (202 wrists) participated in the present study, but 14 wrists were excluded due to steroid injection or wrist surgery. In addition, 2 patients ( 3 wrists) were excluded due to no US results (Fig. 1). Of the 106 patients, the majority of participants were female $(82.1 \%)$ with a mean age of $53.1 \pm 12.8$ years, ranging from 28 to 89 years. Of the 185 wrists examined, $26.0 \%$ ( 48 wrists), $17.8 \%$ ( 33 wrists), $37.8 \%$ ( 70 wrists), and $18.4 \%$ (34 wrists) were documented by NCS as no, mild, moderate, and severe CTS, respectively. Additionally, inter-rater reliability was explored from 28 patients (48 wrists). The result showed that intraclass correlation coefficients $(2,1)$ was 0.93 (95\% confidence interval, 0.87-0.96).

A mean \pm standard deviation of CSA of the median nerve of no, mild, moderate, and severe CTS was 9.4 \pm 2.1 , $12.0 \pm 2.7,13.8 \pm 4.7$, and $15.4 \pm 4.1$, respectively. A moderate degree of positive correlation between CTS severities and CSA was shown (Spearman rank correlation coefficient=0.56) (Fig. 2). The CSA of the median nerve at the wrist had fair diagnostic abilities for moderate and severe CTS - area under the ROC curve $=0.77$ (95\% CI, 0.70-0.83)

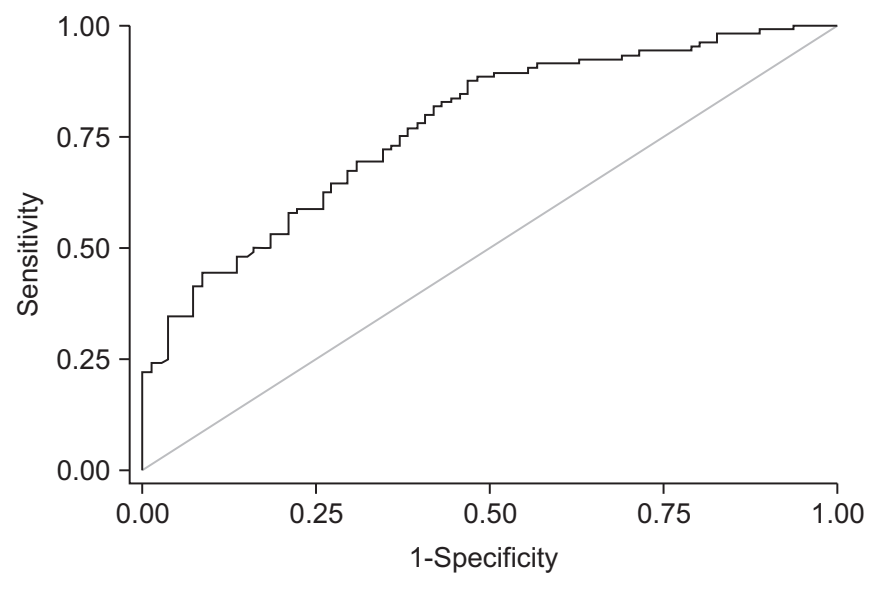

Area under ROC curve $=0.77(95 \% \mathrm{Cl}: 0.70$ to 0.84$)$

Fig. 3. Receiver operator characteristic (ROC) curves with area under the curve of median nerve CSA (cross-sectional area) at wrist, to determine moderate and severe carpal tunnel syndrome. 
Table 1. Diagnostic properties of each different cutoff value

\begin{tabular}{ccccc}
\hline $\begin{array}{c}\text { CSA }\left(\mathbf{m m}^{2}\right) \text { for being } \\
\text { moderate and severe CTS }\end{array}$ & Sensitivity & Specificity & PPV & NPV \\
\hline$\geq 10.0$ & $89.4(81.9-94.6)$ & $48.2(36.9-59.5)$ & $68.9(60.4-76.6)$ & $78.0(64.0-88.5)$ \\
$\geq 11.0$ & $78.9(69.7-86.2)$ & $59.3(47.8-70.1)$ & $71.3(62.1-79.4)$ & $68.6(56.4-79.1)$ \\
$\geq 12.0$ & $62.5(52.5-71.8)$ & $74.1(63.1-83.2)$ & $75.6(65.1-84.2)$ & $60.6(50.3-70.3)$ \\
$\geq 13.0$ & $52.9(42.8-62.8)$ & $80.3(69.9-88.3)$ & $77.5(66.0-86.5)$ & $57.0(47.4-66.3)$ \\
$\geq 14.0$ & $42.3(32.7-52.4)$ & $91.4(83.0-96.5)$ & $86.3(73.7-94.3)$ & $55.2(46.4-63.8)$ \\
$\geq 15.0$ & $34.6(25.6-44.6)$ & $93.8(86.2-98.0)$ & $87.8(73.8-95.9)$ & $52.8(44.3-61.1)$ \\
\hline
\end{tabular}

Values are presented as \% (95\% confidence interval).

CSA, cross-sectional area; PPV, positive predictive value; NPV, negative predictive value.

(Fig. 3). According to ROC curve, an $11.5 \mathrm{~mm}^{2}$ CSA could be the best trade-off between sensitivity $(69.2 \%)$ and specificity (67.9\%). However, its power to rule in or to rule out was insufficient, since PPV and NPV were merely $73.5 \%$ and $63.2 \%$, respectively. A $14 \mathrm{~mm}^{2}$ CSA had sufficient power to rule in moderate and severe CTS, with a specificity of $91.4 \%$ and sensitivity of $42.3 \%$ (Table 1 ). In addition, it showed a post-test probability (PPV) of $86.3 \%$ for a pre-test probability of $56.2 \%$.

\section{DISCUSSION}

The US to measure CSA of the median nerve at the wrist is a useful test to determine moderate and severe CTS. The results of the present study revealed a positive correlation between CSA and CTS severities $\left(r_{s}=0.56\right)$, which was similar to previous studies $[15,16,20]$. The study of Mhoon et al. [20] and Moran et al. [16] reported 0.52 and 0.61 of positive correlation, respectively. However, the primary objective of both these studies differed from the current study. The study of Mhoon et al. [20] was undertaken to establish a cut-off to use US as a screening test of CTS, and suggested CSA of $9 \mathrm{~mm}^{2}$ as the cut-off with a sensitivity of $99 \%$ and specificity of $22 \%$. The study of Moran et al. [16] aimed to determine the cut-off for CTS diagnosis. The study suggested US as the first CTS diagnostic tool, and that CSA of $9.8 \mathrm{~mm}^{2}$ (sensitivity of $92 \%$ and specificity of $45 \%$ ) and $12.3 \mathrm{~mm}^{2}$ (sensitivity of $62 \%$ and specificity of $95 \%$ ) should be used as the cut-off to rule out and to rule in CTS, respectively.

Some patients with electrophysiologically moderate and severe CTS were considered to receive surgical management. A highly sensitive diagnostic test is required to determine the condition. Hence, a $14 \mathrm{~mm}^{2}$ CSA with a sensitivity of $42.3 \%$ and specificity of $91.4 \%$ was preferred, rather than a $13 \mathrm{~mm}^{2}$ CSA having sensitivity of $52.9 \%$ and specificity of $80.3 \%$. Moreover, if the cut-off was increased from $14 \mathrm{~mm}^{2}$ to $15 \mathrm{~mm}^{2}$, there was minimal increase in the specificity and PPV (2.4\% and $1.5 \%$, respectively), but there was a significant drop in sensitivity (7.7\%).

Several studies suggested that the comparison of median nerve CSA between the proximal and distal site, CSA ratio or difference, might increase the diagnostic properties of US [21,22]. Nevertheless, the measurement of CSA at the proximal site showed low reliability. The study of Junck et al. [23] revealed that the intra-rater reliability of measurement of the median nerve CSA at the pronator quadratus were 0.29 for sonographers and 0.49 for radiologists, whereas the intra-rater reliability of measurement of median nerve CSA at carpal tunnel inlet were 0.87 for sonographers and 0.86 for radiologists. Therefore, median nerve CSA at wrist alone was used in the present study.

The present study had several strengths. Firstly, this constituted a prospective study with consecutive enrollment; the US examiner was blinded, and all examined wrists were suspected of having CTS to avoid spectrum bias, which was found in case-control studies. Secondly, the inter-rater reliability was excellent, which influenced the quality of the US information. Finally, both NCS and US were performed on the same day to prevent data error from disease progression.

On the other hand, some limitations were also observed. Firstly, the number of male participants in the present study was small (17.9\% of participants). There were evidences that the CSA of median nerve at the wrist was significantly different between male and female; however, this evidence was observed in healthy subjects, 
and not in CTS patients [24-26]. Next, the US examiner had only 2 years of experience in neuromuscular US, which possibly affected validity of the US results. Lastly, the participants of this study were Thai people, and hence the results could differ in a population of different ethnicity, such as a European population.

In conclusion, US might be used to determine the electrophysiological severities of CTS. Patients with $\geq 14 \mathrm{~mm}^{2}$ of median nerve CSA had a very high probability to be moderate and severe CTS. However, if median nerve, CSA is less than $14 \mathrm{~mm}^{2}$, patients still required NCS to determine CTS severities.

\section{CONFLICT OF INTEREST}

No potential conflict of interest relevant to this article was reported.

\section{ACKNOWLEDGMENTS}

We would like to thank Assoc. Prof. Jariya Boonhong, MD for her contribution in the design of the study. The present study was funded by Phramongkutklao Research funding.

\section{REFERENCES}

1. Atroshi I, Gummesson C, Johnsson R, Ornstein E, Ranstam J, Rosen I. Prevalence of carpal tunnel syndrome in a general population. JAMA 1999;282:153-8.

2. American Association of Electrodiagnostic Medicine, American Academy of Neurology, and American Academy of Physical Medicine and Rehabilitation. Practice parameter for electrodiagnostic studies in carpal tunnel syndrome: summary statement. Muscle Nerve 2002;25:918-22.

3. Rempel D, Evanoff B, Amadio PC, de Krom M, Franklin G, Franzblau A, et al. Consensus criteria for the classification of carpal tunnel syndrome in epidemiologic studies. Am J Public Health 1998;88:1447-51.

4. Wong SM, Griffith JF, Hui AC, Lo SK, Fu M, Wong KS. Carpal tunnel syndrome: diagnostic usefulness of sonography. Radiology 2004;232:93-9.

5. Cartwright MS, Walker FO. Neuromuscular ultrasound in common entrapment neuropathies. Muscle Nerve 2013;48:696-704.
6. Bayrak IK, Bayrak AO, Kale M, Turker H, Diren B. Bifid median nerve in patients with carpal tunnel syndrome. J Ultrasound Med 2008;27:1129-36.

7. Nakamichi K, Tachibana S. Unilateral carpal tunnel syndrome and space-occupying lesions. J Hand Surg Br 1993;18:748-9.

8. Padua L, Liotta G, Di Pasquale A, Granata G, Pazzaglia $\mathrm{C}$, Caliandro $\mathrm{P}$, et al. Contribution of ultrasound in the assessment of nerve diseases. Eur J Neurol 2012;19:4754.

9. Cartwright MS, Hobson-Webb LD, Boon AJ, Alter KE, Hunt $\mathrm{CH}$, Flores $\mathrm{VH}$, et al. Evidence-based guideline: neuromuscular ultrasound for the diagnosis of carpal tunnel syndrome. Muscle Nerve 2012;46:287-93.

10.Ziswiler HR, Reichenbach S, Vogelin E, Bachmann LM, Villiger PM, Juni P. Diagnostic value of sonography in patients with suspected carpal tunnel syndrome: a prospective study. Arthritis Rheum 2005;52: 304-11.

11. Mohammadi A, Afshar A, Etemadi A, Masoudi S, Baghizadeh A. Diagnostic value of cross-sectional area of median nerve in grading severity of carpal tunnel syndrome. Arch Iran Med 2010;13:516-21.

12. Altinok T, Baysal O, Karakas HM, Sigirci A, Alkan A, Kayhan A, et al. Ultrasonographic assessment of mild and moderate idiopathic carpal tunnel syndrome. Clin Radiol 2004;59:916-25.

13. Tai TW, Wu CY, Su FC, Chern TC, Jou IM. Ultrasonography for diagnosing carpal tunnel syndrome: a metaanalysis of diagnostic test accuracy. Ultrasound Med Biol 2012;38:1121-8.

14. Nakamichi K, Tachibana S. Ultrasonographic measurement of median nerve cross-sectional area in idiopathic carpal tunnel syndrome: diagnostic accuracy. Muscle Nerve 2002;26:798-803.

15. Ghasemi M, Abrishamchi F, Basiri K, Meamar R, Rezvani M. Can we define severity of carpal tunnel syndrome by ultrasound? Adv Biomed Res 2015;4:138.

16. Moran L, Perez M, Esteban A, Bellon J, Arranz B, del Cerro M. Sonographic measurement of cross-sectional area of the median nerve in the diagnosis of carpal tunnel syndrome: correlation with nerve conduction studies. J Clin Ultrasound 2009;37:125-31.

17. LeBlanc KE, Cestia W. Carpal tunnel syndrome. Am Fam Physician 2011;83:952-8.

18. Robinson LR, Micklesen PJ, Wang L. Strategies for an- 
alyzing nerve conduction data: superiority of a summary index over single tests. Muscle Nerve 1998;21: 1166-71.

19. Stevens JC. AAEM minimonograph \#26: the electrodiagnosis of carpal tunnel syndrome. American Association of Electrodiagnostic Medicine. Muscle Nerve 1997;20:1477-86.

20. Mhoon JT, Juel VC, Hobson-Webb LD. Median nerve ultrasound as a screening tool in carpal tunnel syndrome: correlation of cross-sectional area measures with electrodiagnostic abnormality. Muscle Nerve 2012;46:871-8.

21. Klauser AS, Abd Ellah MM, Halpern EJ, Siedentopf C, Auer T, Eberle G, et al. Sonographic cross-sectional area measurement in carpal tunnel syndrome patients: can delta and ratio calculations predict severity compared to nerve conduction studies? Eur Radiol 2015;25:2419-27.

22. Klauser AS, Halpern EJ, De Zordo T, Feuchtner GM, Arora R, Gruber J, et al. Carpal tunnel syndrome assessment with US: value of additional cross-sectional area measurements of the median nerve in patients versus healthy volunteers. Radiology 2009;250:171-7.

23. Junck AD, Escobedo EM, Lipa BM, Cronan M, Anthonisen C, Poltavskiy E, et al. Reliability assessment of various sonographic techniques for evaluating carpal tunnel syndrome. J Ultrasound Med 2015;34:207788.

24. Wanitwattanarumlug B, Varavithya V. Evaluating the mean cross-sectional area (CSA) of median nerve by use of ultrasound in Thai population. J Med Assoc Thai 2012;95 Suppl 12:S21-5.

25. Bathala L, Kumar P, Kumar K, Shaik A, Visser LH. Normal values of median nerve cross-sectional area obtained by ultrasound along its course in the arm with electrophysiological correlations, in 100 Asian subjects. Muscle Nerve 2014;49:284-6.

26. Sassi SA, Giddins G. Gender differences in carpal tunnel relative cross-sectional area: a possible causative factor in idiopathic carpal tunnel syndrome. J Hand Surg Eur Vol 2016;41:638-42. 